\title{
SELECTING A SYNTHESIS MODE IN THE ANALYTIC HIERARCHY PROCESS
}

\author{
Ido Millet \\ Penn State Erie \\ Erie, PA 16563-1400 \\ ixm7@psuvm.psu.edu \\ Thomas L. Saaty \\ University of Pittsburgh \\ Pittsburgh, PA 15260 \\ saaty@vms.cif.pitt.edu
}

\begin{abstract}
This paper proposes revised guidelines for the selection of a synthesis mode in the AHP and modifications to software implementations of the methodology. The proposed software changes include adding a Distributive mode to absolute measurement models (Rating models), presenting the raw preference scores from the Ideal synthesis mode, and requiring the user to select a synthesis mode only when that choice has a significant impact on the results of the model.
\end{abstract}

\section{Introduction}

At the heart of the controversy surrounding the Analytic Hierarchy Process [Saaty, 1977] lies the thorny issue of rank reversal [Dyer, 1990a \& 1990b; Harker \& Vargas, 1990; Saaty, 1990]. The "Orthodox" camp claimed that rank reversal is a correct reflection of real life and provided convincing examples of such situations. The "Reformist" camp provided equally convincing examples where such reversals should not take place. Furthermore, the "Reformist" camp proposed new synthesis procedures within the Analytic Hierarchy Process (AHP) that prevent such rank reversals [Belton and Gear, 1983].

While the dust hasn't settled yet, the 1994 Third International Symposium on the AHP reflected the emergence of an integrative view. The consensus is that both camps are correct and that the AHP should support two different synthesis modes: one which allows for rank reversals and one which prevents it. The acceptance of both views is evident in Saaty's article in Interfaces [1994a] which directs us to select one of two synthesis modes: "Distributive" or "Ideal". Both of these synthesis modes have been implemented in the more recent versions of "Expert Choice," the leading software implementation of the AHP.

This bodes well for the continued success of the AHP since it is uniquely positioned to address both types of decision making contingencies. On the one hand the AHP accommodates situations where rank reversals should be avoided. On the other hand, the AHP also supports situations where rank reversals can and should occur [Tyszka, 1983; Freeman et al., 1990; Forman, 1992] in contrast to the axiomatic foundations of utility theory. As such, the AHP offers a practical decision making methodology that is unmatched in its completeness:

The preference reversal phenomenon which is inconsistent with the traditional statement of preference theory remains....No alternative theory currently available appears to be capable of covering the same extremely broad range of phenomenon." [Grether and Plott, 1979].

The bad news is that the availability of two acceptable synthesis modes requires that we select one each time we use the AHP. But, how should we decide which synthesis mode suits our situation best? That is the topic of this paper.

While selecting the wrong synthesis mode would probably make little difference in the results, it is important we reduce user anxiety over this process. Saaty and Vargas [1993] showed empirically that in most cases the choice of synthesis mode doesn't affect the ranks computed for the alternatives. However, without clear guidelines, users of the AHP frequently feel uncomfortable and insecure about the choice of a synthesis mode. 
This paper begins by reviewing the current guidelines for selecting a synthesis mode within the AHP. After identifying problems with the current selection guidelines, new guidelines are proposed and demonstrated through examples. The paper then calls for the uniform application of these guidelines by adding the option of a Distributive synthesis mode to absolute measurement (Rating) models. The paper concludes with a few suggestions for future research and for modifications to software implementations of the AHP.

\section{Problems with Current Selection Guidelines}

When addressing the question of when to use the Distributive synthesis mode, the manual for Expert Choice provides the following guidelines:

To allow your preference for alternatives to be dependent on what other alternatives are available (and, correspondingly, how many there are) you should select the distributive mode which normalizes the weights of the alternatives during synthesis. The Distributive mode is used for resource allocation and for planning where the ranks of the alternatives are affected by the other alternatives. In general, if one is certain that the alternatives, in fact, are different and well separated in their characteristics, the Distributive mode should be used.

In a recent article in Interfaces, Saaty [1994a] provides the following guidelines for selecting the Distributive mode:

It is used when there is dependence among the alternatives... We need to account for such dependence in allocating resources, in voting and in distributing resources among the alternatives.

These guidelines are somewhat vague and perhaps even misleading. It is not clear why the Distributive mode should be used for resource allocation, for planning, and for voting. For example, in allocating resources to four possible R\&D projects, the ideal mode may well be used. While the allocation formula may be based on normalizing the final scores of the alternatives, the procedure for deriving those scores may well be the Ideal synthesis mode. Similarly, in deciding which president to vote for, an individual may well use the ideal mode. "Planning" is a diverse domain, so the reference to it is vague. We see no reason why the ideal mode can't be used in many planning problems.

The recommendation of using the distributive mode when the alternatives are "different and well separated in their characteristics" is most troubling, particularly since it is used in reverse when prescribing the ideal mode. When addressing the question of when to use the Ideal synthesis mode, the manual for Expert Choice provides the following guidelines:

The Ideal mode is useful when distinction between alternatives is not clear, and you do not want copies or near-copies to affect the outcome.

This could be misleading since the degree to which the alternatives are similar to each other should not influence the choice of synthesis mode. The only reason that this issue found its way into the current guidelines seems to be that it is easy to demonstrate unwelcome rank reversals through the introduction of an identical alternative. It should be clear however that the same methodological issues exist even when you introduce alternatives that are not identical. The choice of a synthesis mode should depend on the nature of the decision making problem -- not on how similar the alternatives are.

Saaty [1994a] directs us to use the Ideal mode when we want to "obtain the single best alternative regardless of what other alternatives there are." The emphasis on "single" in this guideline may be too restrictive since it is possible to provide examples where the Ideal mode should be used when the objective is to select multiple alternatives.

\section{Proposed Selection Guidelines}

\section{Contrasting the Two Synthesis Modes}

The main difference between the two synthesis modes is that with the distributive mode the preference for an alternative under each criterion depends on its performance compared to all other alternative. This is what Saaty refers to as the "dependence" issue in his guidelines [1994]. In contrast, the ideal mode determines the preference for an altemative under each criterion by comparing its performance to a fixed 
have to manage individual designations of synthesis types for each criterion node directly above the alternatives.

\section{Descriptive versus Normative Considerations}

The choice of synthesis mode should be influenced by the purpose of the model. While the Ideal mode may be the correct model, the Distributive model may better predict actual choice behavior (and vice versa). For example, when voting for a president, an individual may be advised to use an Ideal model. After all, once the president is selected, all other candidates are irrelevant. However, the Distributive mode may better predict the actual behavior of individual voters.

The complexity of recommending a synthesis mode is further increased when we realize that the choice of a synthesis mode may depend on the characteristics of the decision maker. For example, automobile reliability may be a performance issue for one person who is simply concerned about the nuisance of car repair, but it may be a dominance issue for another person who is concerned about not coming across as spending more time on repair than his 3 best friends do.

It would be interesting to investigate how individual characteristics as well as decision context influence the choice of synthesis mode and the accuracy of these models in predicting actual choice behaviors. For example, if men are more concerned with dominance, would this be reflected in actual choice behavior or in tendencies to select the Distributive mode over the Ideal?

\section{Presentation of the Synthesis Results}

When using the Distributive mode the final weights synthesized for each alternative sum up to 1.00 since the weights under each node are always normalized. In the Ideal synthesis mode though, the weights of the alternatives are not normalized. This means that an altemative can score a perfect 1.00 if it is the best alternative under all the criteria.

Yet, software implementations of the AHP, such as "Expert Choice," take the liberty of normalizing the results of the Ideal synthesis mode before presenting them to the user. For example, four alternatives with Ideal synthesis scores of $0.1,0.4,0.6$, and 0.9 would be presented with final weights of $0.05,0.2,0.3$, and 0.45 respectively. This may seem like a harmless exercise in re-scaling, but we lose valuable information in the process. This is because the initial scores reflect how close the alternatives were to performing best on all criteria. For example, the alternative with a synthesis score of 0.9 is quite close to performing best on all alternatives. After normalization, that information is lost.

The solution is simple -- software implementations of the AHP should not normalize the final scores derived by the Ideal synthesis mode.

\section{Management By Exception}

So far we assumed that the user should select a synthesis mode before using the model. This is also the way "Expert Choice" implements the AHP. We would like to suggest a different approaich -- let the computer generate the results using both synthesis modes and then approach the choice question in the context of its impact.

The empirical research conducted by Saaty and Vargas [1993] indicates that typically there are only minor differences in the results produced by the two synthesis modes. This implies that we should require the decision maker to select a synthesis mode only if the results diverge beyond a given set of exception criteria.

For example, the software may allow the decision maker to indicate that the Ideal mode should be used as a default as long as the change from one synthesis mode to the other has very little impact on the final weights of the alternatives. A "significant" impact should probably be defined through user controlled parameters such as percent and absolute differences in the scores computed for each alternative by the two synthesis modes.

If the results diverge by more than the exception levels, the software should present the decision maker with a side-by-side comparison of the results, and request a choice of a synthesis mode. As an advanced option, the software may also flag situations where due to conducting sensitivity analysis, a divergence exception has occurred. This approach will simplify the use of the AHP since in most cases the decision maker will not be bothered with the choice of a synthesis mode. 


\section{Conclusions}

As a practical and popular methodology the AHP has acquired a life of its own. Thus, it is important to review software implementations of the AHP and existing guidelines for its use. This paper provides such a critical review on the issues surrounding the choice of a synthesis mode within the AHP.

This critical review suggests revised guidelines for the selection of a synthesis mode and modifications to software implementations of the AHP. The proposed software changes include adding a Distributive mode to absolute measurement models (Rating models), presenting the raw preference scores from the Ideal synthesis mode, and requiring the user to select a synthesis mode only when that choice has a significant impact on the results of the model.

\section{References}

Belton, V. \& T. Gear (1983), "On a Short-coming of Saaty's Method of Analytic Hierarchies," Omega, (December, 1983), 228--230.

Dyer, S. James (1990a), "Remarks On The Analytic Hierarchy Process," Management Science, 36, 249--258

Dyer, S. James (1990b), "A Clarification of 'Remarks On The Analytic Hierarchy Process'," Management Science, 36, 274--275.

Foreman, E. H. (1992), "Multicriteria Prioritization in Open and Closed Systems," George Washington University, working paper.

Freeman, K. M., A. R. Pratkanis, and P. H. Farquhar (1990), "Phantoms as Psychological Motivation: Evidence for Compliane and Reactance Processes," working paper.

Grether, D. M. and C. R. Plott (1979), "Economic Theory of Choice and the Preference Reversal Phenomenon," The American Economic Review, 69, No. 4, 623--638.

Harker, T. Patrick \& Luis G. Vargas (1990), "Reply to 'Remarks On The Analytic Hierarchy Process'," Management Science, 36, 269--273.

Saaty, T. L. (1977), A scaling method for priorities in hierarchical structures," Journal of Mathematical . Psychology, 15, 59--62.

Saaty, L. Thomas (1990), "An Exposition Of The AHP In Reply to the Paper 'Remarks On The Analytic Hierarchy Process'," Management Science, 36, 259--268.

Saaty, T. L. and Vargas, L. G. (1993), "Experiments on Rank Preservation and Reversal in Relative Measurement," Mathematical and Computer Modelling, 17, No. 4/5, 13--18.

Saaty, L. Thomas (1994a), "How to Make a Decision: The Analytic Hierarchy Process," Interfaces, 24, (1994a), 19--43.

Saaty, L. Thomas (1994b) Fundamentals of Decision Making and Priority Theory with the Analytic Hierarchy Process, RWS Publications, Pittsburgh, PA.

Tyszka, Tadeusz (1983), Human Decision Making, edited by Lennart Sjoberg, Tadeutsz Tyszka and James A. Wise . 